\title{
Service Quality Assurance Mechanisms for P2P SIP VoIP
}

\author{
Xiaofei Liao, Fengjiang Guo, and Hai Jin \\ Services Computing Technology and System Lab \\ Cluster and Grid Computing Lab \\ School of Computer Science and Technology \\ Huazhong University of Science and Technology, Wuhan, 430074, China
}

hjinahust.edu.cn

\begin{abstract}
Recently P2P (Peer-to-Peer) has been proposed to improve the scalability of the traditional VoIP (voice over IP) systems. However, P2P makes VoIP service unreliable because $\mathrm{P} 2 \mathrm{P}$ networks probably scale up and the service nodes are very likely to fail or leave the P2P networks when VoIP service is offering. To deal with this issue, we propose a service quality assurance mechanism. The whole proposition includes a relay overlay algorithm and a dynamic relay algorithm. In the scheme, VoIP system can select the successor of the failed relay nodes without adding extra backup nodes. The simulation results demonstrate that our algorithms maintain $70 \%$ voice dialogs correctly in the presence of relay nodes failure with the delay remaining below $150 \mathrm{~ms}$. The results also show that the system architecture is feasible and scalable.
\end{abstract}

Keywords: Voice over IP, Service quality, Backup links, Dynamic switching.

\section{Introduction}

Voice over IP (VoIP) system is gradually replaying traditional PSTN (Public Switched Telephone Network) telephone and becoming the key pattern of voice transportation in NGN (Next Generation Network) due to its low cost, high flexibility and ability of integrating multimedia information.

The P2P technology enhances VoIP system performance, but also brings some problems. P2P overlay network is open and extensible, but also has great differences and dynamics. The characteristic makes $\mathrm{P} 2 \mathrm{P}$ networks unable to meet the requirements of VoIP system which is a kind of real-time application. When two VoIP nodes communicate in this network, the system often has quality of service problems.

The unreliability of service is a common issue in traditional VoIP systems, and it is mainly due to the breakdown or departure of the service node. Traditional service quality improvement mechanisms use RSVP (Resource Reservation Protocol) in application-layer, and MPLS (Multi-protocol Label Switching) in physical layer for improving service quality. The main disadvantages of those solutions are as follows:

- High costs: the cost of hardware and maintenance is increased, because it is based on the redundant mechanisms.

- Poor scalability: the scalability of the system is subject to the constraints of the server capacity, and the increasing number of the user means that the service of the system is needed to be upgraded constantly. 
- Performance bottleneck: those solutions do not take the property of P2P network into account. The delay of restoration does not meet the requirement of real-time systems.

On the other hand, in currently VoIP system, the more popular solution for traversing the firewall is to add a relay node between the users. However, due to property of the relay node, the system often has quality of service problems because the service node may leave the relay overlay. In that case the VoIP call is likely to fail.

The relay overlay consists of relay nodes and relay servers. The relay node is selected by relay server in VoIP system. In our VoIP system, the user applies to relay server for appropriate node before initiating a call. The unreliability of this relay service has extreme impact on the call process, because the call will be forced to be suspended once the failure or departure of caller's relay node.

As mentioned above, how to design an affective mechanism to find a successor of the failed relay node is still a problem. We propose a solution of a dynamic relay algorithm based on ICE (Interactive Connectivity Establishment) protocol. The main idea is that a lightweight redundant mechanism for relay server is adopted. If two users need to relay when communicating, one can use the relay server find the appropriate node to relay. We will explain the relay-node finding mechanism between two relay servers in the paper in detail. Before the call is initialized, the callee does not hold the information of the caller. It will select the relay node for accomplishing ICE process. At the same time, when the VoIP system makes the call with that relay node, it can dynamically switch the relay node which can provide better service. The callee automatically detects the voice quality and sends RE-INVITE (a command in SIP protocol) request. Then the callee is staying on the phone with the caller in the case of poor voice quality. In this case, the callee who holds the caller's information such as the position of its relay server could use a better relay node detecting algorithms.

In this paper we study the framework of P2P-SIP system and relay detecting algorithms. The problem proposed is how to design service quality assurance mechanism for P2P-SIP VoIP system to take full advantage of the properties of relay overlay to achieve high reliability and low investment VoIP system.

The rest of this paper is organized as follows. Section 2 discusses the related work. The detail system architecture is described in section 3. Section 4 describes our simulation and presents the performance evaluation. We conclude this work in section 5.

\section{Related Work}

In this section, we give a brief overview of Skype, a real VoIP system, and related technologies including an application-layer relay algorithm.

\subsection{Skype Relay Calls}

Skype is the most popular P2P-based commercial VoIP system, which works around NAT (Network Address Translation) and firewall issues by routing calls through the machine of another Skype user with unrestricted connectivity to the Internet.

Skype client (SC) maintains a list of other Skype peers called a host cache (HC) [4]. Other Skype peers list is empty when a SC is running for the first time, and is built during the lifetime of a Skype client. Then, a SC can contact nodes in the host cache 
(HC). Additionally, a SC has a built-in list of about seven default Skype nodes, which are called bootstrap nodes and are used to help peers join the Skype overlay for the first time after installation.

After joining successfully, a Skype client establishes a TCP connection with another Skype user or a super node (SN). There are two types of nodes in the Skype overlay: super node $(\mathrm{SN})$ and ordinary node. Both $\mathrm{SN}$ and ordinary node run the same Skype software. SNs are responsible for detecting online SCs, and transmitting signaling messages between SCs [3]. To establish a call, a SC searches for the callee machine. If they successfully contact with each other, they can directly exchange media data with the callee machines. We can select a node as a relay node (RN) and the call can be routed. A RN is a SN, which has sufficient bandwidth to relay a voice or a video call.

A key aspect of Skype's robust connectivity is its ability to traverse NAT and firewalls by routing a video or voice call through one or more Skype relays. The network conditions between the caller and callee machines are configured so that they are forced to use a RN.

The success rate of relay calls depends on the network conditions, the presence of a host cache, and caching of the callee's reachable address. From our experiments, we have found that Skype relay mechanism can be further improved. Because the Skype uses private protocol and the most important problem is how to guarantee the relay node steady and dependable.

\subsection{Application-Layer Relay Algorithm}

The application-layer relay algorithm is a one-hop relay algorithm. It sets an overall server in relay overlay. This server is called relay server and manages other peers. The peers in the overlay keep the information of other nodes in the network and divide them into some parts. This information of other nodes will be stored in a ring-structure called Meridian [1]. It sets the radius of every ring according to the delay. The peers ping each other and get the delay among them, and put the other nodes in its rings. These nodes are used when the system needs a relay call.

While one peer wants to join the network, it will ask the relay server for some node lists according to its network environment. After detecting the peers in these lists, the new node can initialize its own rings. It will update their delay information by using timer. The nodes send update packets to some of the peers in their rings. According to the approach, the node can update the peers in their rings.

When users need a relay call, it sends the search packet including the information of another user. The search algorithm will send the result back to the user who requests the relay call. The process is as follows. First, the source peer sends the search packets to some of its neighbors in its inner ring. The packets include IP address of the destination node. The nodes who receive the search packets continue to transfer the packet to the node in its own neighbors until it finds the destination node. By defining survival time of these search packets, it can reach some nodes so that there will be more opportunity to find the relay.

The application-layer relay algorithm is used in our early system which is called as Cutephone. But the relay node plays important role, and its network condition is crucially important for the call completion and service quality. Unfortunately, the relay call is used to solve the problem when users are behind NAT and firewall. The callee does not know the caller's information before initializing a call in most call cases. The relay path it selects may not be the best option. 


\section{Design and Architecture}

There are two levels in our VoIP system architecture, the signal overlay and the media relay overlay, as shown in Figure 1. The upper level is signal overlay, which consists of super node client (SN-C). The SN-C is responsible for signal transferring. The lower level is media relay overlay, and it consists of relay servers and relay nodes. Their respective functions are described in the following sections. A UE (the VoIP client) could be standard traditional SIP clients or our own P2P-SIP client called Cutephone. Cutephone is more flexible than tradition SIP clients by adding some functions to support ICE (Interactive Connectivity Establishment).

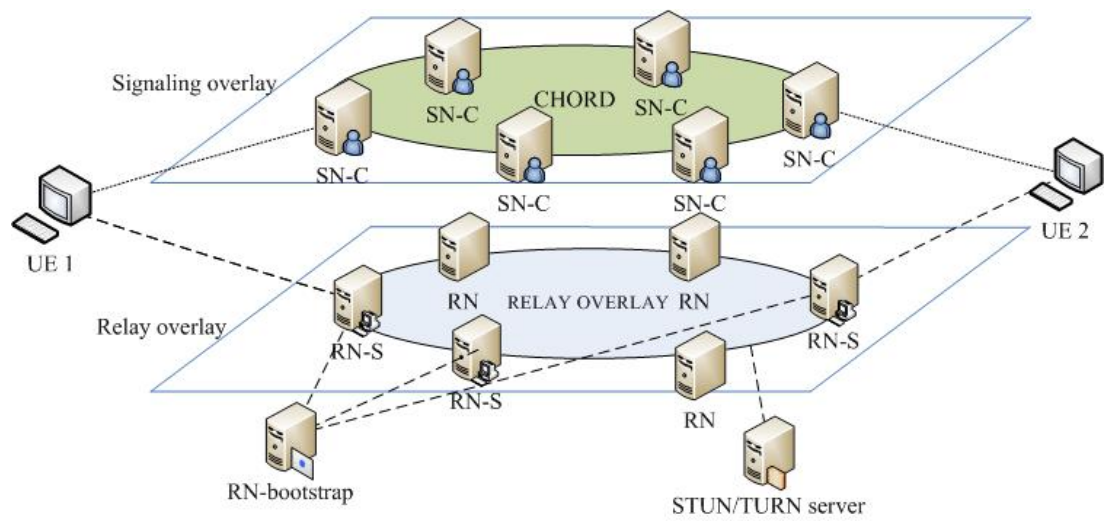

Fig. 1. P2P VoIP system structure

\subsection{Design Principle}

The system consists of Super Node Client (SN-C), Relay Node Server (RN-S), and Relay Node (RN). They play different roles in the VoIP system.

SN-C combines the functions of SIP register server, SIP redirect server, and SIP agent server. Actually SN-C has two layers. One is the tradition SIP layer, called SIP server, and the other is the $\mathrm{P} 2 \mathrm{P}$ overlay layer. $\mathrm{P} 2 \mathrm{P}$ overlay is maintained by the $\mathrm{SN}-\mathrm{C}$ mainly for user orientation and user information publishing, the specific algorithm of $\mathrm{P} 2 \mathrm{P}$ overlay is Chord protocol.

$\mathrm{RN}-\mathrm{S}$ manages the peers in relay overlay. It keeps the information of other nodes in the network and divides them into some parts according to the delay among them. This information of other nodes will be kept in a Meridian rings.

RN transfers RTP (Real-time Transport Protocol) packets between UEs if necessary. Besides, RN helps UEs which involves NAT traversal problem. The UEs use ICE to traverse NAT and firewall. The relay node's address is one of the candidate addresses in ICE process. ICE details will be described below.

The service quality assurance mechanisms should meet the following principles:

- The system should backup the relay call link before the call initializing. Otherwise the delay of the whole relay node switching cannot be ignored. 
- The relay detecting algorithm should be divided into two different parts. As mentioned above, the relay node which is selected before may not be the best option. So the relay detecting algorithm must be distinguished before and after first relay call.

- The RN-S plays an important role in relay call. Its stability will have influence on the ICE and the relay call procedures. So we should consider the RN-S reliability.

\subsection{System Architecture Overview}

According to the above principle, we adopt redundant communication mechanisms in RN-S management of UEs. The whole architecture of relay overlay is shown in Figure 2.

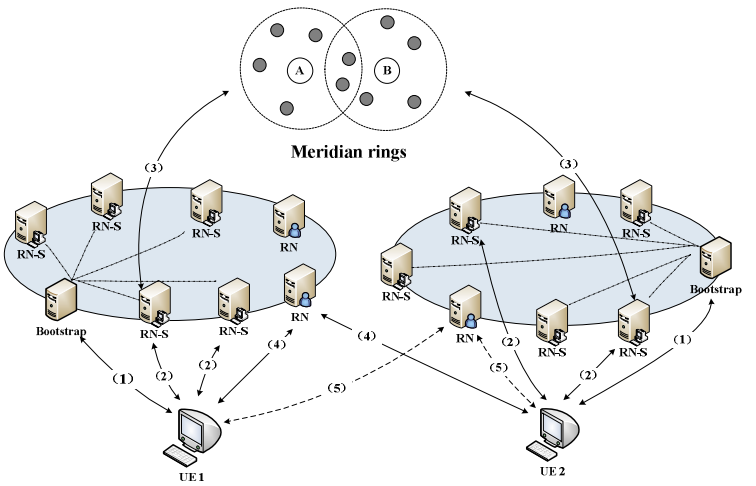

Fig. 2. Relay call procedure

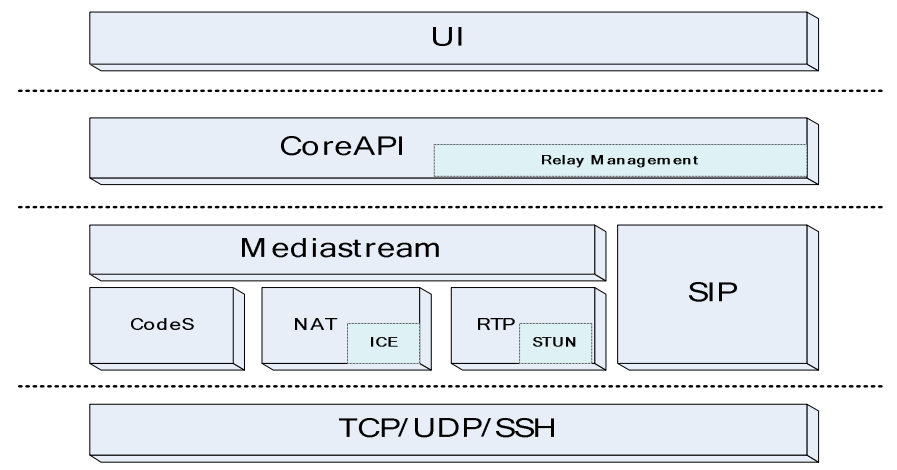

Fig. 3. Cutephone application modules

In Cutephone, the above relay call process and service quality assurance mechanism are replaced by relay overlay structure. The software is four-layer structure, as shown in Figure 3. The top layer is UI (User Interface) module, which analyzes UE's operation command and calls the functions provided by lower layer. 
The Core API module provides the relay management function which can decide the call modes. There are two kinds of call modes, direct and relay call, respectively. The Media stream module uses codes module to encode and decode the audio/video data. The NAT module uses ICE to traverse NAT and firewall. The RTP and STUN module defines a standardized packet format for delivering audio and video over IP networks and traverse them. The SIP module handles SIP request and response.

\subsection{RN-S Register}

As shown in Figure 4, the process that UEs register in the relay overlay network is the same as that UEs register in the P2P network according to a bootstrap node.

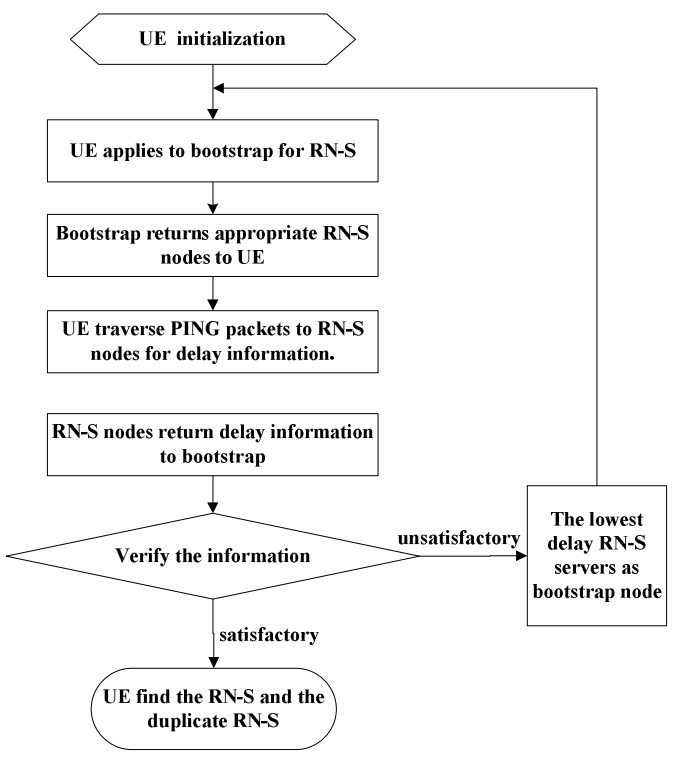

Fig. 4. RN-S registration

Specific registration process is as follows:

1) When a UE starts up, it will register in relay overlay with the bootstrap node in the list of bootstrap it maintains.

2) UE sends requests to bootstrap node for its RN-S nodes. Then the bootstrap node returns appropriate $\mathrm{RN}-\mathrm{S}$ nodes according to UE's geographic information.

3) UE tests the delay between itself and the RN-S nodes returned by bootstrap node. Then it will check if the delay values can meet its requirements.

4) If satisfied, the registration is finished and the lowest delay nodes will be UE's RN-S node. Otherwise, it will serve as bootstrap node and take step 1.

The actual UE registration in relay overlay involves more than one bootstrap node. The multi-bootstrap nodes will ensure UE's registration in the relay overlay successfully. 


\subsection{Redundancy Detecting}

The process that UEs request its RN-S nodes through relay overlay network is not different from ordinary detecting algorithm if the RN-S response acknowledgement message is available. However, it may be failed because of the unreliable characteristic of the overlay network. In these cases, UE will send request to its duplicate RN-S, and then wait for the returned relay node, as shown in Figure 5. The timer in our mechanism asks the UEs to see if the relay node server is failed or departed.

After sending request message to the RN-S and waiting for the response, the UEs must finish the process of relay detecting. If the UEs work behind the symmetric NAT, the relay call mode is necessary. Moreover, the relay node is important for candidate address collection and connectivity checks in ICE technology.

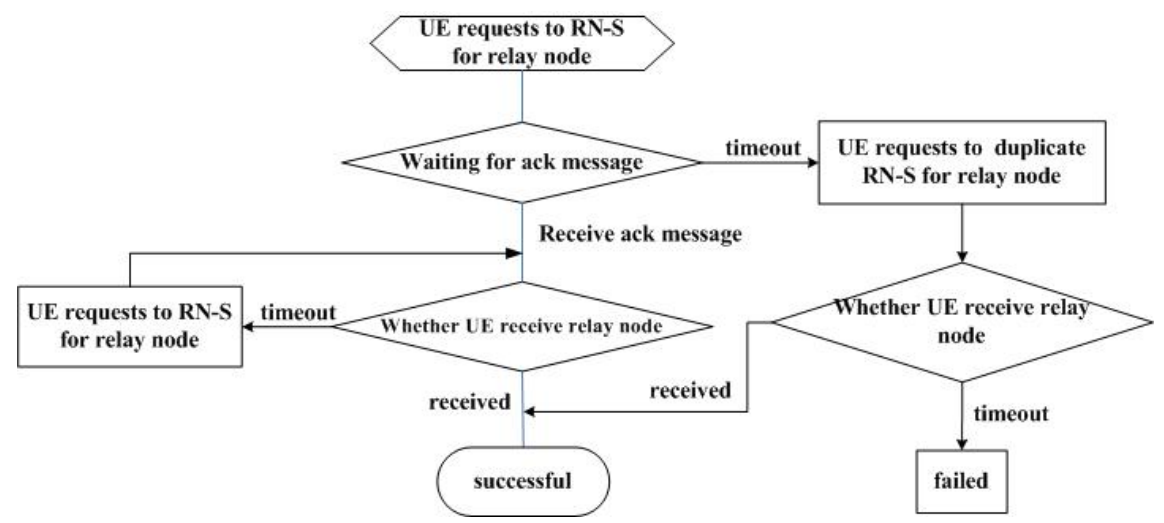

Fig. 5. Redundancy detecting

\subsection{NAT Traversal}

The problem of NAT traversal can be resolved by ICE technology. The IETF (Internet Engineering Task Force) has devised a suite of protocols, namely STUN (Session Traversal Using NAT) [9], TURN (Traversal Using Relay NAT) [10], and ICE [11], to address the limitations of the currently available NAT traversal solutions. Making a call starts by sending a SIP INVITE message with an SDP describing on which IP address and port the application can receive audio and/or video packets. These addresses and ports are known as candidates. Candidates are obtained from any firewall engine and are inserted into the SDP of a SIP INVITE message, which is sent to the callee.

There are 3 types of candidates: local candidate, a local IP address of the client; reflexive or STUN candidates, an IP address of the client's NAT (assuming they are only behind a single NAT); and relay candidate, an address on a relay node that has been allocated by the client.

After gathering the candidates, the caller encodes them in the SIP INVITE message, and sends the message to the callee according to signaling overlay. In response to the SIP INVITE, the callee sends its ICE candidates within the SDP of a SIP provisional response, such as a SIP 183 (Session Progress), to the caller. Once the callee has sent its ICE candidates and the caller receives them, they will start 
performing ICE connectivity checks. At this time, both parties know about their peer's potential transport candidates. Each possible pair of local and remote candidates is formed. A connectivity check is done by sending STUN messages from the local candidate to the remote candidate for each pair, starting with the highest priority candidate pair first. Both parties exchange STUN messages in this way to determine the best possible candidate pair based on that they can communicate. Once a successful message has been sent both ways on a single candidate pair, the connectivity check may stop and media can be sent/received using that candidate pair.

\subsection{Dynamic Relay Algorithm}

The dynamic relay algorithm is based on the ICE protocol (draft-ietf-mmusic-ice-19) and the relay detecting algorithm. It provides a self-testing and failure restoration mechanism. Due to the breakdown or departure of relay node, the relay call service is unreliable. For that reason, the UEs must dynamically switch relay links. In SIP messages, the re-INVITE can modify the dialog and the parameters of the sessions at the same time. Consequently, we use the re-INVITE message and send it to the callee for new relay link establishment.

The other important procedure is the inquiry for a relay node. As we have said, the relay detecting algorithm can be divided into two parts. Before the call is initialized, we use ICE technology to determine the call link. When UEs are likely to switch the relay links, the process of choosing relay nodes is as follows:

Step 1. UE $A$ requests the relay node corresponding to RN-S $A$ and RN-S $B$;

Step 2. for each relay $A i$ in Meridian rings of $\mathrm{RN}-\mathrm{S} A$;

For each relay $B i$ in Meridian rings of $\mathrm{RN}-\mathrm{S} A$;

if $(\mathrm{A} i==B i)$ do return relay $A i$;

Endif;

Else do $B i+1$;

$A i+1$;

Step 3. RN-S $A$ returns the relay node $A i$ to UE $A$, UE $A$ makes it as relay node. Then UE $A$ makes a call start by sending a SIP re-INVITE message with a SDP describing on relay node's IP address and port the application can receive audio and/or video packets.

Algorithm 1. Relay node detecting algorithm before switching

The specific process of dynamic relay algorithm is shown in Figure 6 and the detailed message flow is as follows:

1) SIP-client-A (UE 1) finds the poor quality of audio or video service, according to the parameter such as packet loss ratio and delay.

2) UE 1 requests a new relay node to its relay server (RN-S 1) with the information of UE 2's relay server RN-S 2. According algorithm 1, the RN-S 1 and $\mathrm{RN}-\mathrm{S} 2$ work together and return the appropriate new relay node to UE 1 .

3) UE 1 makes a new call by sending re-INVITE message with a SDP message on new relay node's address and port the application can receive audio and/or video packets.

4) UE 1 transmits STUN packets to new relay node's address and port. On the other hand, UE 2 transmits the RTP packets to the address. 


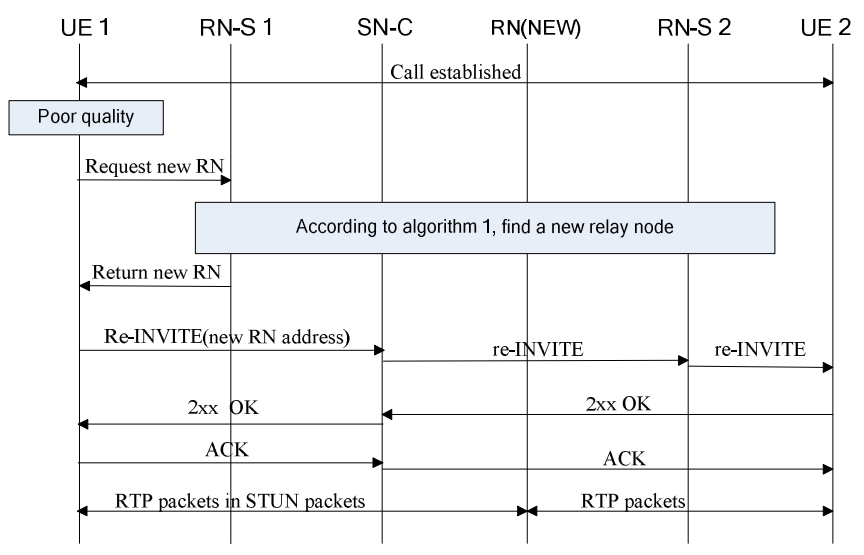

Fig. 6. AAA structure of P2PSIP

Considering the condition that all of the SIP clients are our applications, there may be a problem if they send the re-INVITE message at the same time. UEs must return a 491 error message for the re-INVITE message. The clients who receive a 491 error message will check if it is a caller. Then the caller startup a timer at the average from 2.1 to 4 second, and the callee's timer is from 0 to 2 second.

\section{Performance Evaluations}

\subsection{Evaluation Environment}

We test our service quality assurance mechanism in the NS-2 (Network Simulation Version 2), which is running on a node of the high performance computing cluster in our lab. The node has two dual-processor Intel Xeon CPU and 8GB memory. We adopt the network model of random connecting of Transit AS with Stub AS, which is similar to the real network. With different sets of delay between, we run the system in different network scales to evaluate the performance.

By checking the results of the simulation, we will estimate the re-hit ratio of the relay nodes, the dialog completion ratio, and the latency in our system, which is to test the stability of the relay mechanism.

\subsection{Re-Hit Ratio}

The re-hit ratio is an important indicator of efficiency in our algorithm. The re-hit ratio indicates the probability that system switches relay nodes dynamically. According the algorithm described in section 3 , the relay node is crucial to service quality assurance mechanism. Higher re-hit ratio brings better reliability of relay sub-system.

From section 3, we can see that a higher percentage of RNs in our relay overlay does not imply that more relay calls are requested. We set the number of RN from 4002400.The results of re-hit ratio equals to the successful switching relay link ratio are shown in Figure 7. 


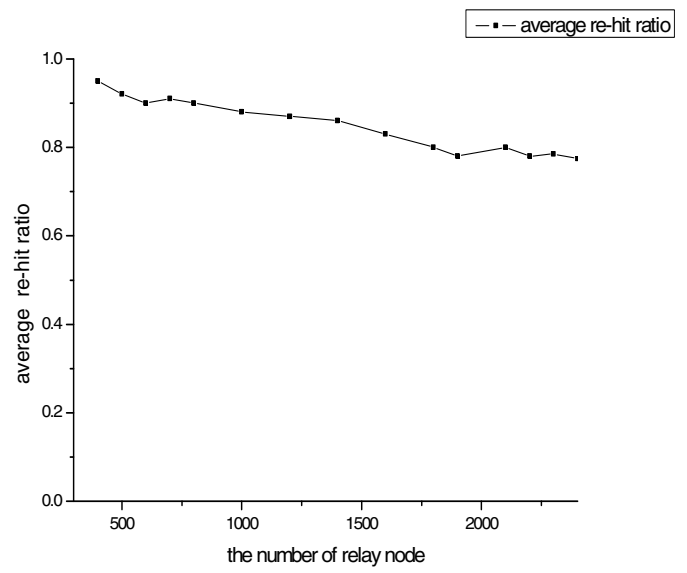

Fig. 7. Re-hit Ratio

As shown in Figure 7, the average re-hit ratio of the system decreases along with the number of RN. The average re-hit ratio refers to the finding rate of fresh relay nodes when UEs switch the relay links under a certain node scale. The algorithm ensures a high re-hit rate in the low node scale. The average re-hit rate is about $90 \%$. But with the expansion of the $\mathrm{RN}$ nodes, the re-hit ratio of the algorithm also decreases, because with the increasing of RN nodes number, the coverage TTL of searching packets will be reduced. But exceeding a certain number of the RN nodes, the hit rate stabilizes at a certain range, which is more than $75 \%$, and proves the stability of the dynamic search algorithm.

\subsection{Dialog Completion Ratio}

The dialog completion ratio is another important indicator of efficiency in system. Dialog completion ratio is defined as the number of successfully completed dialogs in a certain period of time to total dialogs. This value can be used to indicate the quality of VoIP service.

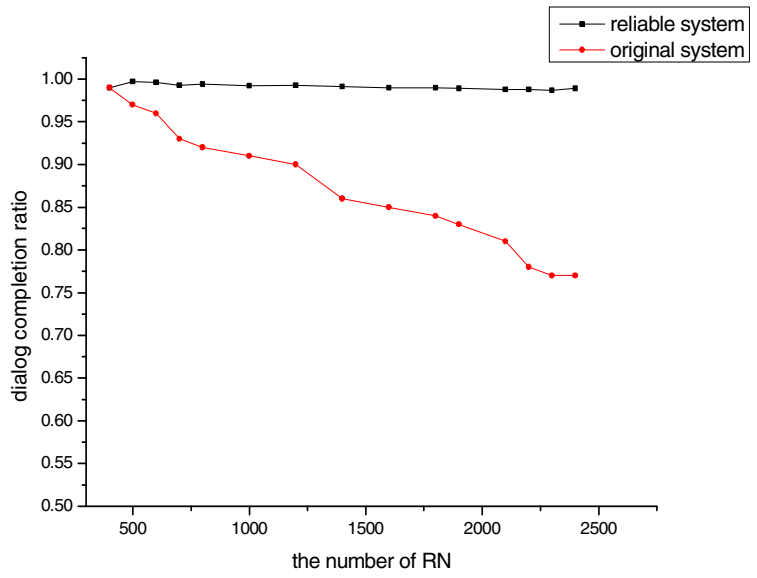

Fig. 8. Dialog completion ratio 
In order to illustrate the unreliability of original VoIP system and the effectiveness of our algorithm, we run simulations for both the original VoIP system and our reliable system, which contains the ICE user software. In our simulation, we uniformly set the disabled nodes among the network and the node disabling time according to the overall simulation time, and we run the overall 1000 sessions with relay call. The results are shown in Figure 8.

The average re-hit ratio of the system decreases with the increasing of the number RN. In spite of the relay node could fail in our system, the algorithm maintains around 98\% dialogs correctly to provide the reliable SIP service. On the other hand, the dialog completion ratio in the original VoIP system decreases along with the number of RN. The main reason of the low ratio may be the performance of the relay detecting algorithm, which also degrades along with the RN node scale. In our system, if the first relay node fails, the UEs will dynamically switch another relay node.

Figure 9 shows the dialog completion ratio changing with the node failure ratio. In the simulation we change the node failure ratio and keep the number of relay nodes about 500. Then we can find the difference between our system and original VoIP system. As shown in Figure 9, the dialog completion ratio decreases with the growth of the relay node failure ratio. The original VoIP system fails even faster, under $30 \%$ when the failure ratio is $50 \%$, while our system is $70 \%$ at the same condition.

\subsection{Latency of Switching Relay Nodes}

The latency of switching relay nodes refers to the average delay of changing a relay node between two UEs under certain situation. We will test the latency from the time when the UE detects the poor quality to the time when one relay node has been decided. The feasibility of the strategy also should be still useful with the expansion of the nodes scale. If the delay value is too big or only be limited within a small node scale, this system can not be used. In our simulation, as the shown in Figure 10, when the system switches relay node, the latency remains about $250 \mathrm{~ms}$. The delay is not satisfied, and the user can feel conversation interrupts, which comes mainly from the delay of ICE process and relay detecting.

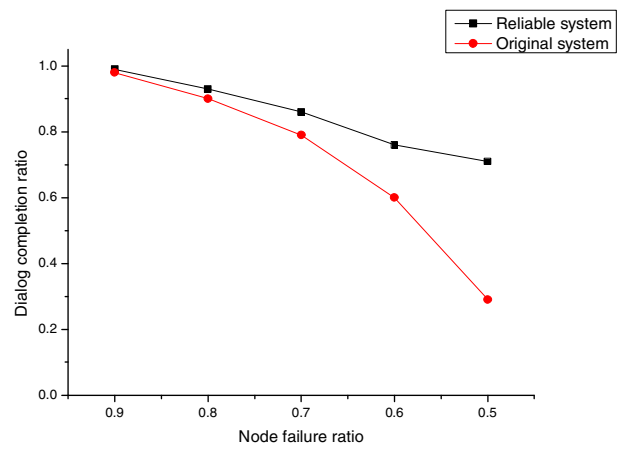

Fig. 9. Dialog completion ratio with node failure ratio 


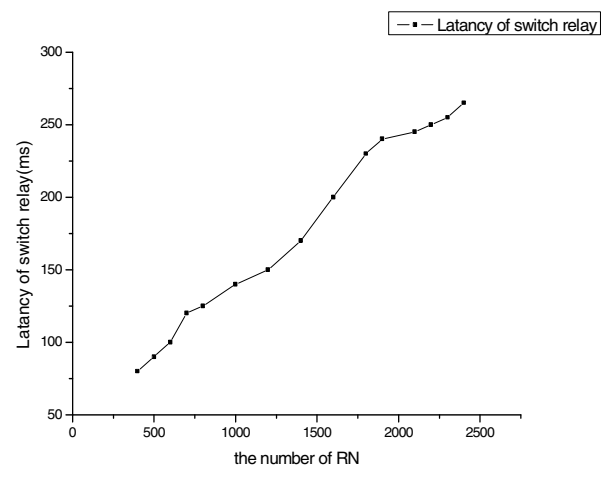

Fig. 10. Latency of switch relay

\section{Conclusions}

In this paper, we propose a service quality assurance mechanism, which maintains the VoIP servers quality in a relay overlay effectively. Simulation results indicate that the system architecture is feasible and scalable.

The whole design includes relay detecting algorithm and dynamic relay algorithm. When the VoIP system builds a call with a relay node, it can dynamically switch the relay node with another one which can provide better service. The callee automatically detects the voice quality and sends re-INVITE request for poor voice quality. Our work provides an initial study on the reliable structure of P2P-SIP VoIP system.

Acknowledgments. This work is supported by Program for New Century Excellent Talents in University under grant NCET-08-0218, China National Natural Science Foundation (NSFC) under grant 60973133, FOK YING TUNG Education Foundation under grant No.122007, the National Science and Technology Major Project of the Ministry of Science and Technology of China under grant No.2010ZX-03004-001-03 and the Fundamental Research Funds for the Central Universities under grant No.No.2010QN013.

\section{References}

1. Wong, B., Slivkins, A., Sirer, E.G.: Meridian: A Lightweight Framework for Network Positioning without Virtual Coordinates. In: The Annual ACM Conference of the Special Interest Group on Data Communication (SIGCOMM 2005), Philadelphia, PA (2005)

2. Singh, K., Schulzrinne, H.: Peer-to-Peer Internet telephony using SIP. In: the 15th International Workshop on Network and Operating Systems Support for Digital Audio and Video (NOSSDAV 2005), pp. 63-68 (2005)

3. Baset, S.A.: An Analysis of the Skype Peer-to-Peer Internet Telephony Protocol. In: INFOCOM 2006, Barcelona, Spain (2006)

4. Jennings, C., Lowekamp, B., Rescorla, E., Baset, S., Schulzrinne, H.: REsource LOcation And Discovery (RELOAD). IETF draft draft-ietf-p2psip-reload-04 (2008) 
5. Baset, S., Gupta, G., Schulzrinne, H.: OpenVoIP: An Open Peer-to-Peer VoIP and IM System. In: ACM SIGCOMM 2008 (demo), pp. 517-517 (2008)

6. Hardie, T.: Pointers for Peer-to-Peer overlay networks, nodes, or resources. IETF Draft draft-hardie-p2psip-p2p-pointers-00 (2008)

7. Jiang, X.F., Zheng, H.W.: Service extensible P2P protocol. IEFT Draft draft-jiang-p2psipsep-01 (2008)

8. Song, H., Zheng, H., Jiang, X.: Diagnose P2PSIP overlay network failures. IETF Draft draft-zheng-p2psip-diagnose-02 (2008)

9. Rosenberg, J., Mahy, R.: Session Traversal Utilities for NAT. IETF STUN draft behaverfc3489bis-10 (2008)

10. Behave, W.G., Rosenberg, J.: Obtaining Relay Addresses from Simple Traversal Underneath NAT. IETF TURN draft behave-turn-04 (2008)

11. Rosenberg, J.: Interactive Connectivity Establishment (ICE): A Protocol for Network. IETF ICE draft mmusic-ice-18 (2007)

12. Address Translator (NAT) Traversal for Offer/Answer Protocols

13. Guha, S., Daswani, N.: An Experimental Study of the Skype Peer-to-Peer VoIP System. In: IPTPS 2006 (2006)

14. Xie, H., Reng, Y.: A Measurement based Study of the Skype Peer to Peer VoIP Performance. In: IPTPS 2007, Bellevue, WA (2007)

15. Fei, T., Tao, S., Guerin, R.: How to select a good alternate path in large peer-to-peer system. In: IEEE INFOCOM 2006 (2006)

16. Shansi, R., Lei, G., Zhang, X.: ASAP: an AS-Aware Peer-Relay Protocol for High Quality VoIP. In: The 26th IEEE International Conference On Distributed Computing System (2006)

17. Shu, T., Kuai, X., Antonio, E.: Improving VoIP quality through path switching. In: IEEE INFOCOM 2005 (2005) 